\title{
X-ray spectrometer for anomalous scattering experiments: tests of a graphite mosaï and a germanium monocrystal as analyzers
}

\author{
M. Bessière, S. Lefebvre, M. Lemonnier, F. Bley* and S. Lequien** \\ LURE (CNRS/CEA/MEN), Bat 209d, 91405 Orsay cedex, France \\ * LTPCM, ENSEEG, UA 29 du CNRS, BP. 75, 38402 Saint Martin d'Hères, France \\ ** ESRF, BP. 220, 38043 Grenoble cedex, France
}

\begin{abstract}
Anomalous X-ray scattering provides a useful way for improving the contrast between different atoms present in multi-element samples.

To take full advantage of this technique, the detector has to have a good energy resolution in the diffracted beam to separate the elastic scattering signal from the inelastic one due to fluorescence or resonant Raman scattering (RRS). Solid state detectors are usually used, but the energy resolution is limited to about $150 \mathrm{eV}$ which does not allow the separation RRS from elastic scattering. Ice and Sparks [1] described a mosaic crystal spectrometer which resolves this problem. We have adapted their setup on our beam line D23A at LURE-DCI but we have encountered a loss of resolution when the Bragg planes of the sample are inclined. The shape of the diffracted beam incoming on the position-sensitive detector depends strongly on the inclination of the diffracting planes; this explains the loss of resolution which is increased in our case by the large horizontal incoming beam size.

Alternatively, we recommend the use of a perfect crystal as an analyzer coupled with a $0 \mathrm{~d}$ detector which gives a very good resolution $(\approx 10 \mathrm{eV})$ without modification of resolution when Bragg planes of the sample are inclined in $\chi$. Furthermore this kind of spectrometer allows a high counting rate so that it is possible to measure close to Bragg peaks (clustering studies, for example) and even Bragg peaks. The loss of efficiency is not dramatic considering the high brightness of third generation synchrotron radiation and is more than compensated by the improved signal to noise ratio.
\end{abstract}

\section{Introduction:}

The tunability of X-Ray synchrotron radiation allows one to take advantage of the drastic variation of atomic scattering factors near $\mathrm{K}$ or $\mathrm{L}$ absorption edges and then to achieve anomalous X-ray scattering. This technique has been applied to the study of long and short range order in ternary alloys, modulated structure, local order in amorphous binary alloys, structure of multilayers, etc... It has its own problems: energy stability of the incident beam, exact knowledge of the real and imaginary terms $\mathrm{f}^{\prime}$ and $\mathrm{f}^{\prime \prime}$ of the structure factor, experimental determination of the scale factor, and separation of the elastic scattering signal from the inelastic one due to fluorescence or resonant Raman scattering (RRS) [2].

Unfortunately, this latter signal is enhanced when the incident X-ray energy is close to an absorption-edge energy and some instrumental efforts are dedicated to the separation of the different signals. Solid state detectors are usually used but their energy resolution is limited to around $150 \mathrm{eV}$ which does not allow to remove RRS from elastic scattering. An attractive solution is the use of a mosaic crystal spectrometer [1]. The advantages are a good resolution $(\approx 20 \mathrm{eV}$, principaly depending on the source size), a good efficiency ( $40 \%$ compared with nearly $100 \%$ for solid state detectors), and a collection of a large energy band $(\approx 300 \mathrm{eV})$. 
We present here tests of such a spectrometer performed on beam line D23A at LURE-DCI and some difficulties we have encountered.

\section{A mosaic crystal spectrometer}

\subsection{General description}

A detailed description of this kind of spectrometer is given in reference [1]. In a parafocusing geometry, mosaic crystals disperse the energy spectrum from the source (sample) along a line collected by a linear-position-sensitive detector (PSD). X-rays penetrate the mosaic crystal until they encounter a mosaic block oriented to allow diffraction (the 200 reflection of a flat graphite crystal is used here). Only rays which deviate from the central ray by less than one half the angular width of the mosaic are diffracted.

The energy bandpass is obtained by differentiating the Bragg law : $\Delta \mathrm{E} / \mathrm{E}=\operatorname{cotg} \theta \Delta \theta$, where $\Delta \theta$ (angular acceptance) is either equal to the mosaic spread if the crystal is too large or is determined by the length $\mathrm{L}$ of the crystal $(\Delta \theta=\mathrm{L} \sin \theta / \mathrm{F}$, where $\mathrm{F}$ is the sample-graphite crystal distance). For our set-up with $\mathrm{F}=400 \mathrm{~mm}$ and a mosaic spread of $0.4^{\circ}$, the energy bandpass is $200 \mathrm{eV}$ at $8 \mathrm{keV}$. The rays of different energies inside the bandpass are focused onto the PSD (sagitally parafocusing technique) according to a spatial distribution $\mathrm{SD}$ given by $\mathrm{SD}=\Delta \mathrm{E} / \mathrm{F} 2 \Delta \theta=\mathrm{E} \operatorname{cotg} \theta / 2 \mathrm{~F}(40 \mathrm{eV} / \mathrm{mm}$ at $8 \mathrm{keV}$ in our case).

The energy spectra of the diffracted beam are encoded by using a PSD. We have used a "homemade" linear Xe gas filled detector with a spatial resolution better than $100 \mu \mathrm{m}$.

\subsection{Tests of energy resolution}

D23A beam line at LURE-DCI is equipped with a double-crystal fixed-exit monochromator (Si 111) where the second crystal is sagitally focusing. The sample was mounted on a Huber 4-circle goniometer described elsewere [3].

The first test was performed by selecting an incoming energy $(\mathrm{Ei}=8365 \mathrm{eV})$ exciting the $\mathrm{X}$-ray fluorescence of a $\mathrm{Ni}$ powder (figure 1). The measured energy resolution is around $25 \mathrm{eV}$. A relevant comparison can be done with the energy spectrum recorded in the same conditions with a $\mathrm{Si}(\mathrm{Li})$ solid state detector (figure 2). The $\mathrm{Ni} \mathrm{K} \beta$ fluorescence line cannot be separated from the elastic one, whereas this is achieved with the spectrometer. The $\mathrm{Ni} \mathrm{K}_{\alpha}$ fluorescence line is not seen in the energy spectrum delivered by the spectrometer due to the small bandpass of the graphite used $(260) \mathrm{eV}$ ). But we can observe it if the incoming angle on the graphite crystal is adjusted.

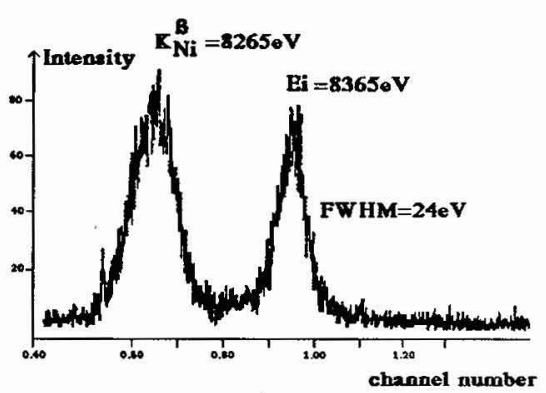

Fig. 1: energy spectrum achieved with the graphite crystal spectrometer in the tail of a Ni powder 111 Bragg peak : Ei $=8365 \mathrm{eV}$.

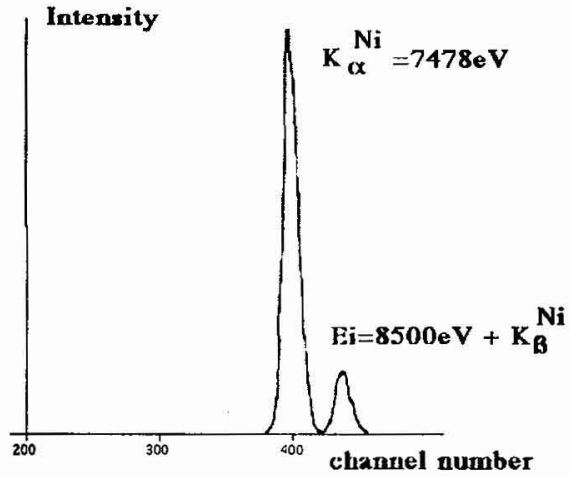

Fig. 2 the same as figure 1 with a $\mathrm{Si}(\mathrm{Li})$ solid state detector. 
Figure 3 displays a second test where the energy is selected $20 \mathrm{eV}$ below the $\mathrm{KNi}_{\mathrm{Ni}}$ threshold. In this way the $\mathrm{M}_{\mathrm{I}}, \mathrm{M}_{\mathrm{II}}, \mathrm{M}_{\mathrm{III}} \mathrm{RRS}$, which occur at $111 \mathrm{eV}, 68 \mathrm{eV}$ and $66 \mathrm{eV}$ respectively below Ei [2], are excited. The separation of the whole RRS from the elastic signal is clearly achieved.

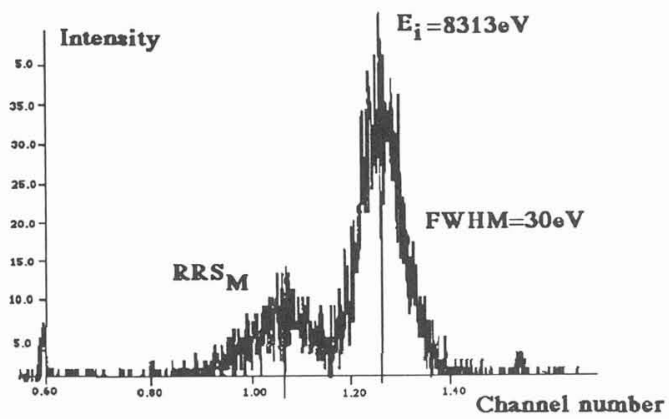

Fig. 3: energy spectrum obtained with the graphite crystal spectrometer at the tail of a $\mathrm{Ni}$ powder 111 Bragg peak : $\mathrm{Ei}=$ $8313 \mathrm{eV}$.

Considering these encouraging results, we have recorded a 220 Bragg peak of a flat Ge single crystal. The (220) planes are parallel to the crystal surface (zero inclination angle $\chi$ ). Figure 4 a shows the energy spectrum with only one line fitting with the elastic energy $E \mathrm{i}=8991 \mathrm{eV}$. The energy resolution $(30 \mathrm{eV})$ is in good agreement with that deduced from the Ni powder test.

On the other hand when the crystal is such oriented to diffract on (311) Bragg planes, the inclination angle becomes $\chi=31^{\circ} 78$ and then the energy spectrum is spread resulting in a worse energy resolution $(\approx 130 \mathrm{eV}$, figure $4 \mathrm{~b})$.
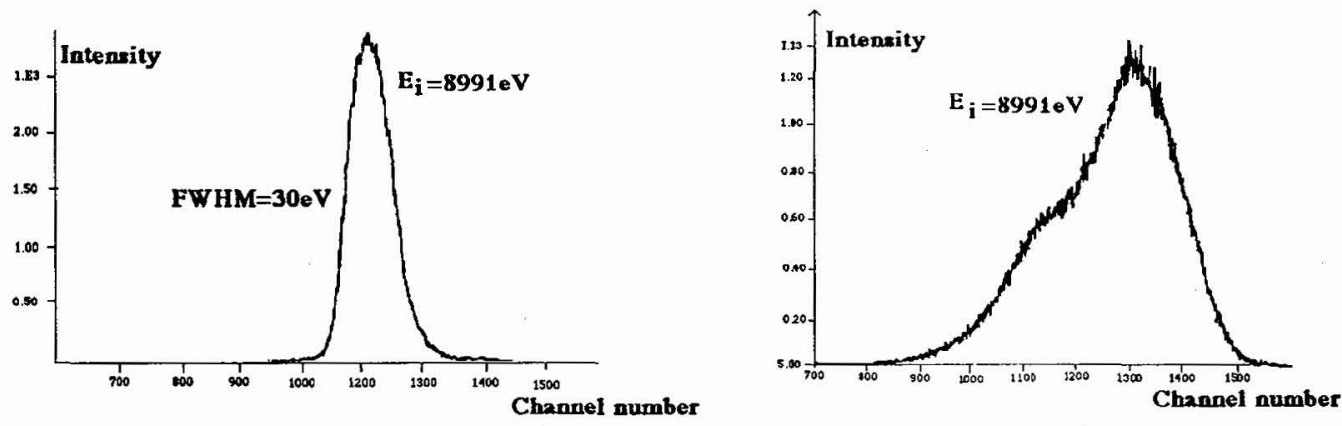

Fig.4: energy spectrum made with the graphite crystal spectrometer for a Ge single crystal as a sample:

a) 220 Bragg peak with $\chi=0^{\circ}$ b) 311 Bragg peak $\chi=31^{\circ} 78$.

\section{Discussion}

The variation of the energy resolution with the inclination angle can be understood when we look at the diffracted beam shape for the two $\chi$ positions (figure 6 ): the beam footprint on the detector wire is broader when $\chi$ increases. 
Geometric considerations which are detailed in ref. [4] demonstrate the energy resolution is directly linked to the beam footprint. For $\chi=0^{\circ}$ the vertical beam size $h$ determines it, as when $\chi \neq 0^{\circ}$ the horizontal size 1 must be taken into account and the beam footprint spreads on the detector wire with a width $2 * 1 * \cos \theta * \tan \chi$. So it yields to a loss of energy resolution.

The use of a narrow horizontal beam by focusing drastically onto the detector wire would decrease this effect as it has been calculated by ray tracing simulations. In the same way the beam delivered by insertion devices at new generation sources should improve it. In any event, the variation of energy resolution versus the inclination angle will not be completely suppressed. Moreover to separate the elastic signal from the inelastic one, a deconvolution of all the signals at each measured point must be performed [5] and the variation of the energy position of the Compton scattering with the reciprocal position makes this more difficult. The data analysis is therefore non-trivial.

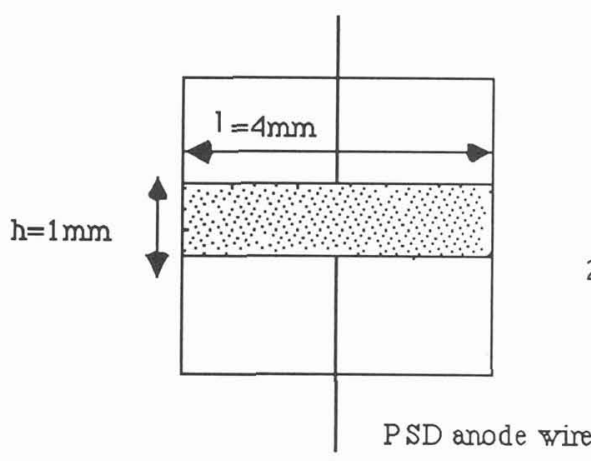

(a)

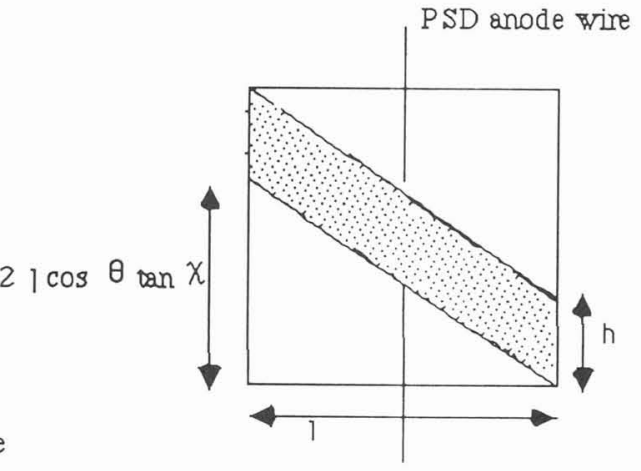

(b)

Fig 6: View with an X-ray camera of the beam footprint after Bragg reflection on a Ge single crystal as a sample: a) $220 \mathrm{Bragg}$ peak with $\chi=0^{\circ}$; b) $311 \mathrm{Bragg}$ peak with $\chi=31^{\circ} 78$.

Another way to build a spectrometer with a good energy resolution is the use of a perfect crystal coupled with a Od detector. We have already tested this solution by using a Ge (111) crystal and a scintillator detector [6]. The energy resolution is the intrinsic width of the relevint Bragg reflection $(\approx 10 \mathrm{eV}$ for 111 reflection in our case).

We have recorded $(\mathrm{Ei}=9094 \mathrm{eV})$, the diffuse scattering of Co.3Pt alloy annealed close to the temperature of the order-disorder transformation and compared it to that obtained with a solid state detector. With this latter set-up some corrections are required to only obtain the elastic signal which is not the case for a perfect crystal spectrometer. It indeed rejects all the inelastic signals, especially the Compton scattering from $\sin \theta / \lambda=0.1$. As an example figure 7 shows for example the high symetry line 100-300 where the 200 Bragg peak is not displayed. The 100 and 300 short range order maxima in reciprocal space are experimentaly found with the same intensity in Laue unit in accordance with the L 12 structure. This was done without any correction but at the detriment of the intensity which is 200 times lower than that recorded with a solid state detector. 


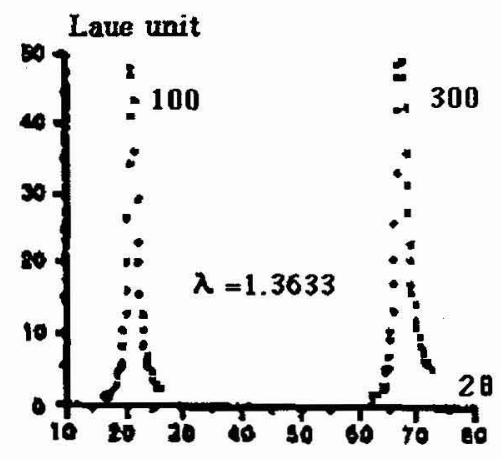

Fig 7: Diffuse scattering of a $\operatorname{Co} 3 \mathrm{Pt}$ single crystal annealed close to the orderdisorder transition temperature.

\section{Conclusion}

Due to the variation of the energy resolution with the inclination angle of the sample, the mosaic crystal spectrometer is not suitable for D23 beam line at DCI.

More investigations may be carried out with a narrower horizontal beam delivered by new generation synchrotron sources. Nevertheless a solution with a perfect crystal as analyzer will always work. This kind of spectrometer gives a very good resolution $(\approx 10 \mathrm{eV})$ without modification of the energy resolution when Bragg planes of the sample are inclined. Coupled also with the new generation of $0 \mathrm{~d}$ detector as avalanche photodiodes, it allows a high counting rate so that it is possible to measure close to Bragg peaks (for example to study clustering) and even Bragg peaks. The loss of efficiency is not so dramatic considering the high brigthness of the third generation synchrotron source (ESRF, APS, SPRING-8, SOLEIL,...) and is more than compensated by the expected improvement of the signal to noise ratio.

\section{Reference}

[1] Ice G.E. and Sparks C.J., NIM A291 (1990) 110-116.

[2] Sparks C. J., Phys. Rev. Lett. 33 (1974) 262-264.

[3] Elkaim E., Lefebvre S., Kahn R., Berar J.F., Lemonnier M. and Bessière M., Rev. Sci. Instrum. 63 (1992) $988-991$.

[4] Lee W. K. and Macrander A., NIM A319 (1992) 158-162.

[5] Reinhard L., Robertson J. L., Moss S.C., Ice G.E., Zschack P., Sparks C.J., Phys. Rev. B 45 (1992) 2662-2676.

[6] Bessière M., Lefebvre S., Elkaim E. and Calvayrac Y., LURE Activity Report (1987-1989) 330. 